\title{
Triptolide inhibits vascular endothelial growth factor-mediated angiogenesis in human breast cancer cells
}

\author{
HUANTAO LIU ${ }^{1}$, LUBING TANG ${ }^{2}$, XIAOYAN LI $^{1}$ and HUIYING LI ${ }^{3}$ \\ ${ }^{1}$ Department of Breast Surgery, Qilu Hospital of Shandong University, Jinan, Shandong 250012; \\ ${ }^{2}$ Department of Breast Surgery, Ningbo Women and Children's Hospital, Ningbo, Zhejiang 315012; \\ ${ }^{3}$ Department of Anesthesiology, The Second Hospital of Shandong University, Jinan, Shandong 250012, P.R. China
}

Received September 20, 2016; Accepted May 15, 2017

DOI: $10.3892 /$ etm.2018.6200

\begin{abstract}
Triptolide has been demonstrated to induce tumor cell apoptosis. However, the role of triptolide in breast cancer angiogenesis remains unclear. The present study aimed to investigate the function of triptolide in breast cancer and the molecular mechanisms underlying this. The results revealed that triptolide could significantly decrease the expression of vascular endothelial growth factor A (VEGFA) in Hs578T and MDAMB231 breast cancer cells. Furthermore, human umbilical vein endothelial cells were used to perform tube formation and bromodeoxyuridine incorporation assays, which demonstrated an antiangiogenic effect of triptolide. In addition, the effect of triptolide in vivo was examined in a xenograft mouse model, which determined that VEGFA, cluster of differentiation 31 and anti-proliferation marker protein Ki67 expression in tumor sections was decreased in the triptolide treatment group compared with the control group. Western bolt analysis was performed to investigate the phosphorylation of extracellular signal-related kinase (ERK) $1 / 2$ and RAC- $\alpha$ serine/threonine-protein kinase after triptolide treatment, and it's effect on hypoxia inducible factor (HIF)1- $\alpha$ expression. The results demonstrated that triptolide suppressed ERK1/2 activation and HIF1- $\alpha$ expression. Furthermore, overexpression of HIF1- $\alpha$ could partially abrogate the inhibitory effect of triptolide on VEGFA expression. These results suggest that triptolide inhibits breast cancer cell angiogenesis in vitro and in vivo through inhibiting the ERK1/2-HIF1- $\alpha$-VEGFA axis.
\end{abstract}

\section{Introduction}

Breast cancer is a common malignancy in women, and has high morbidity and mortality rates. There are $\sim 250,000$ new

Correspondence to: Dr Lubing Tang, Department of Breast Surgery, Ningbo Women and Children's Hospital, 339 Liuding Street, Ningbo, Zhejiang 315012, P.R. China

E-mail: donald_roo@sina.cn

Key words: triptolide, vascular endothelial growth factor, angiogenesis, breast cancer, hypoxia inducible factor $1-\alpha$ cases of breast cancer and $\sim 40,000$ breast cancer-associated mortalities annually in the USA (1). The risk factors for breast cancer remain relatively unclear, although it has been reported that multiple factors, including hormone levels, family heredity and obesity, may influence the initiation and development of breast cancer $(2,3)$. Due to its high heterogeneity, breast cancer is characterized into several subgroups according to genome alterations. However, the diagnosis and treatment for breast cancer requires improvement. To improve survival rates and reduce recurrence, increased understanding of the molecular mechanisms underlying breast tumor progression is necessary.

Angiogenesis serves an essential role in tumor growth and metastasis (4). In breast cancer, tumor cells may disseminate to distant organs or tissues, including the lung and bone, through blood vessels formed from endothelial cells (5). In addition, these new blood vessels formed deliver blood and nutrients for continued tumor growth. Angiogenesis is stimulated by angiogenic factors secreted by the tumor, including vascular endothelial growth factor A (VEGFA), platelet-derived growth factor (PDGF), fibroblast growth factor (FGF) and angiopoietins $(6,7)$. Thus, these factors could be therapeutic targets for breast cancer.

Triptolide has been widely recognized as an anti-inflammatory, antioxidant and antiproliferative compound $(8,9)$. Triptolide is extracted from the herb Tripterygium wilfordii and has been used as an antibiotic (9). A previous study reported that triptolide was beneficial for the treatment of monocytic and myelocytic leukemia (10). Triptolide induces tumor cells apoptosis by activating the caspase cascade and inhibiting Wnt/ $\beta$-catenin signaling (11). Furthermore, triptolide can covalently bind to and inhibit the transcription factor II human protein (12). These observations suggest that triptolide has potential efficacy for the treatment of breast cancer. Indeed, Owa et al has demonstrated that triptolide exhibits cytotoxicity against breast cancer cells in vitro and in vivo through causing lysosomal-mediated programmed cell death (13). However, the function of triptolide in angiogenesis remains unclear.

The present study examined the effects of triptolide on breast cancer cells, particularly angiogenesis. This revealed that triptolide could inhibit VEGFA expression and breast cancer cell proliferation. In vivo injection of triptolide inhibited the formation of microvessels. In addition, triptolide inhibited the activation of extracellular signal-related kinase (ERK)1/2 
and the expression of hypoxia inducible factor (HIF)-1 $\alpha$. The overexpression of HIF1- $\alpha$ partially induced VEGFA expression despite continuing triptolide treatment. These results indicate that triptolide has potential for the clinical treatment of breast cancer.

\section{Materials and methods}

Cell culture and reagents. MCF7, MDAMB231, and T47D were obtained from the Type Culture Collection of the Chinese Academy of Sciences (Shanghai, China); SKBR3, Hs578T and BT474 human breast cancer cells were obtained from the American Type Culture Collection (Manassas, VA, USA). Cells were maintained in RPMI-1640 medium (Gibco; Thermo Fisher Scientific, Inc., Waltham, MA, USA) supplemented with $10 \%$ fetal bovine serum (Gibco; Thermo Fisher Scientific, Inc.), $10 \mathrm{U} / \mathrm{ml}$ penicillin and $10 \mathrm{mg} / \mathrm{ml}$ streptomycin. Cells were cultured in a humidified incubator at $37^{\circ} \mathrm{C}$ with $5 \% \mathrm{CO}_{2}$. Triptolide was purchased from Santa Cruz Biotechnology, Inc. (Dallas, TX, USA; cat. no. sc-200122) and dissolved in dimethyl sulfoxide (DMSO). Cells were treated with indicated concentration of Triptolide and cultured in 24, 48 and $72 \mathrm{~h}$ and treated with DMSO as control.

Plasmid and small interfering (si)RNA transfection. Human HIF1- $\alpha$ complementary DNA was amplified using the polymerase chain reaction (PCR) and subcloned into the expression vector pCDNA3.1. The primers used for PCR were as follows: Sense' 5'-CGGGATCCATGGAGGGCG CCG'-3' and antisense' 5'-GCTCTAGACTAAATAATTCCT ACT'-3'. siRNA pools targeting HIF1- $\alpha$ (HSH008831) or VEGFA (HSH018475) were purchased from GeneCopoeia, Inc. (Guangzhou, China). siRNA transfection were performed using Lipofectamine ${ }^{\circledR} 2000$ Transfection reagent (Invitrogen; Thermo Fisher Scientific, Inc.) according to the manufacturer's protocol, which has been described previously (14). For overexpression of HIF1- $\alpha$, the plasmid was transfected in cells using Lipofectamine 2000.

RNA extraction, reverse transcription-quantitative (RT-q)PCR and semi-quantitative PCR. Total RNA was extracted from cells using TRIzol reagent (Invitrogen; Thermo Fisher Scientific, Inc.) according to the manufacturer's protocol. cDNA was synthesized using the PrimeScript RT-PCR kit (Takara Biotechnology Co., Ltd., Dalian, China) according to the manufacturer's protocol and used as a template for qPCR. qPCR was performed on the Applied Biosystems 7500 Fast Real-Time PCR system (Thermo Fisher Scientific, Inc.) using SYBR Green methods $\left(S_{Y B R}{ }^{\circledR}\right.$ Premix Ex Taq ${ }^{\mathrm{TM}}$ II; Takara Biotechnology Co., Ltd.) according to the manufacturer's protocol, which has been described previously (15). The expression levels of the target genes were normalized to GAPDH using the $2^{-\Delta \Delta \mathrm{Cq}}$ method (16). The primers used for qPCR were as follows: VEGFA forward' 5'-AGGGCAGAA TCATCACGAAGT'-3' and reverse' 5'-AGGGTCTCGATT GGATGGC'-3'; and GAPDH forward' 5'-CTGGGCTACACT GAGCAC'-3' and reverse' 5'-AAGTGGTCGTTGAGGGCA AT'-3'. The semi-quantitative real-time PCR was performed using Takara Ex Taq (\#RR001A, Takara Biotechnology Co. Ltd.) with VEGFA primers. The cDNA used was the same. The following thermocycling condition were used: $95^{\circ} \mathrm{C}$ for
$10 \mathrm{sec}, 58^{\circ} \mathrm{C}$ for $15 \mathrm{sec}$ and $72^{\circ} \mathrm{C}$ for $30 \mathrm{sec}$ for 30 cycles. The PCR products were loaded in $1 \%$ agarose gel, stained with ethidium bromide and semiquantification was performed by gray intensity analysis.

Western blotting. Total protein was extracted using radioimmunoprecipitation assay buffer (Sigma-Aldrich; Merck KGaA, Darmstadt, Germany) with added protease inhibitor and phosphatase inhibitor cocktail (Roche Applied Science, Mannheim, Germany. Protein concentration was measured using the bicinchoninic acid assay. For each sample, $60 \mu \mathrm{g}$ of total protein per lane was separated via SDS-PAGE on a $10 \%$ gel and then transferred to a polyvinylidene fluoride membrane. The membranes were blocked with $5 \%$ bovine serum albumin (cat. no. B600036; Sangon Biotech; Shanghai, China) for $1 \mathrm{~h}$ at room temperature and then incubated with primary antibodies at $4{ }^{\circ} \mathrm{C}$ overnight at a 1:1,000 dilution. Then, the membranes were washed with PBS-Tween-20 and incubated at room temperature for $45 \mathrm{~min}$ with horseradish peroxidase (HRP)-conjugated goat anti-rabbit immunoglobulin G secondary antibodies (cat. no. sc-2004; 1:2,000 dilution; Santa Cruz Biotechnology, Inc.). The results were visualized using enhanced chemiluminescence with ECL Western Blotting Substrate (cat. no. 32106; Pierce; Thermo Fisher Scientific, Inc.). GAPDH was used as the loading control. The primary antibodies used were as follows: Anti-phosphorylated (p)-ERK1/2 (cat. no. 4376), anti-ERK1/2 (cat. no. 4695,), anti-p-RAC- $\alpha$ serine/threonine-protein kinase (Akt; cat. no. 4060), anti-Akt (cat. no. 4691) and anti-HIF1- $\alpha$ (cat. no. 14179) (all Cell Signaling Technology, Inc., Danvers, MA, USA) and anti-GAPDH (cat. no. sc-25778; Santa Cruz Biotechnology, Inc.). The quantification of western blot bands was performed using ImageJ software (National Institutes of Health, Bethesda, MD, USA).

Animal experiments. The animal experimental procedures used in the present study were approved by the Animal Care and Use Committee of Shandong University and in accordance with guidelines. The mice were purchased from Shanghai SLAC Laboratory Animal Co., Ltd. (Shanghai, China). A total of $1 \times 10^{6}$ MDAMB231 cells were injected into the mammary fat pads of 128 -week-old female nude mice (weight, $20.21 \pm 1.18 \mathrm{~g}$ ) to produce a mouse model of breast cancer. Mice were housed at $25^{\circ} \mathrm{C}$ and had free access to food and water with a 14-h light/10-h dark cycle. These mice were then divided into two groups ( $n=6 /$ group), the untreated control group and the triptolide treatment group. A total of 15 days after xenotransplantation of the cells, $1 \mathrm{mg} / \mathrm{kg}^{-1}$ triptolide was injected into the tumors 3 times per week for a total of 4 weeks or when the tumor size reached $\sim 1.5 \mathrm{~cm}$. Then the mice were sacrificed, and the tumors were extracted and processed for immunohistochemistry.

Immunohistochemistry. Tumor tissues were collected 8 weeks after the last triptolide injection, and underwent $4 \%$ formalin fixation overnight at $4{ }^{\circ} \mathrm{C}$ and paraffin embedding. The specimens were then sliced into $4-\mu \mathrm{m}$-thick sections. Then, the sections were deparaffinized in xylene and rehydrated in alcohol. After antigen retrieval at $\sim 100^{\circ} \mathrm{C}$ for $3 \mathrm{~min}$ in citrate buffer, the sections were placed in $3 \% \mathrm{H}_{2} \mathrm{O}_{2}$ to quench 
A

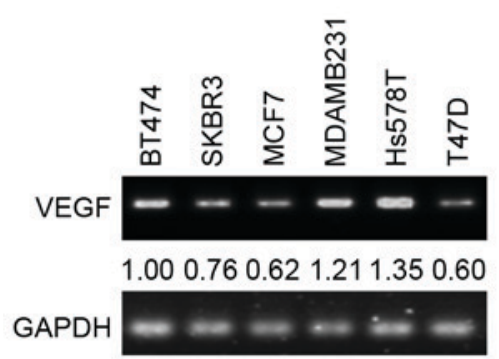

B

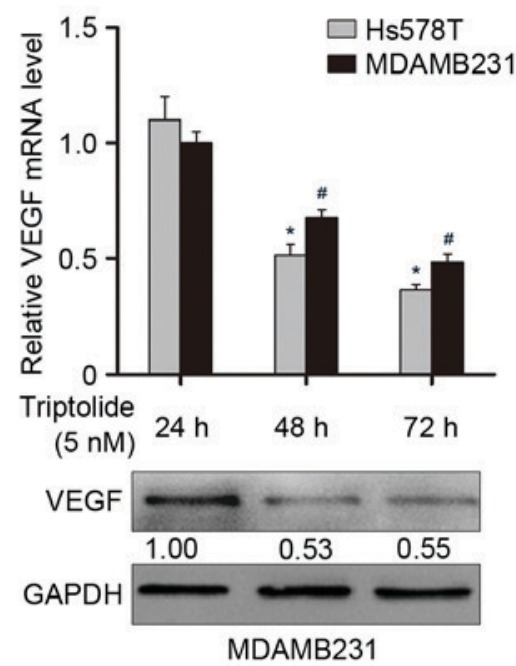

C

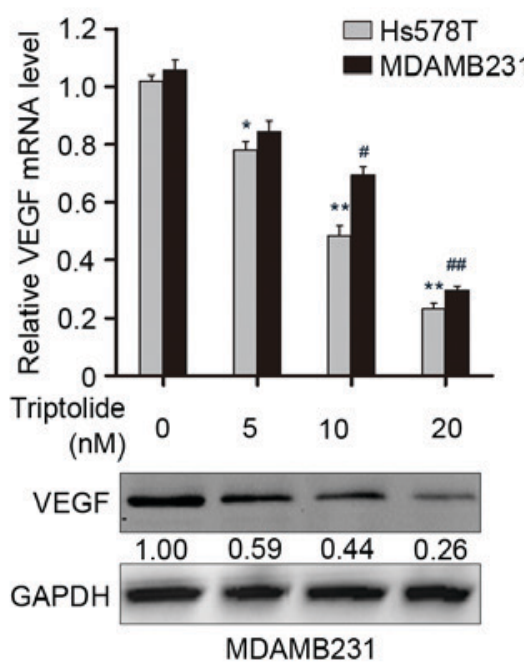

Figure 1. Triptolide suppresses the expression of VEGFA in vitro. (A) VEGFA mRNA expression levels in six breast cancer cell lines were determined using RT-qPCR analysis. GAPDH was used as the internal control. (B) Hs578T and MDAMB231 were treated with $5 \mathrm{nM}$ triptolide for 24,48 and $72 \mathrm{~h}$, and the expression of VEGFA was examined by RT-qPCR (upper panel) and western blot (lower panel) analysis. (C) Cells were treated with dimethyl sulfoxide alone or triptolide $(5,10$ or $20 \mathrm{nM})$, and the expression of VEGFA was examined by RT-PCR (upper panel) and western blot $\left(\right.$ lower panel) analysis. ${ }^{*} \mathrm{P}<0.05,{ }^{* *} \mathrm{P}<0.01$ vs. Hs578T cells at $24 \mathrm{~h}$ or $0 \mathrm{nM}$ triptolide treatment, respectively; ${ }^{\#} \mathrm{P}<0.05$, ${ }^{\# \#} \mathrm{P}<0.01$ vs. MDAMB231 cells at $24 \mathrm{~h} 0 \mathrm{nM}$ triptolide treatment, respectively. VEGFA, vascular endothelial growth factor A; RT-qPCR, reverse transcription-quantitative polymerase chain reaction.

endogenous peroxidase activity and samples were blocked with 5\% BSA (Sangon Biotech) at room temperature for $30 \mathrm{~min}$. Primary antibodies were incubated with the sections overnight at $4^{\circ} \mathrm{C}$. Subsequently, the sections were washed with PBS and incubated with HRP-labeled secondary antibodies for $30 \mathrm{~min}$ at $37^{\circ} \mathrm{C}$, followed by a further wash. The antibody signals were visualized using 3'-diaminobenzidine and counterstained with hematoxylin at room temperature for $2 \mathrm{~min}$. Sections were then observed under a light microscope. At least three sections per specimen were stained to confirm reproducibility. The primary antibodies used were as follows: Anti-VEGFA (cat. no. 19003-1-AP; 1:200 dilution), anti-cluster of differentiation (CD)31 (cat. no. 11265-1-AP; 1:200 dilution) and anti-proliferation marker protein Ki67 (Ki67; cat. no. 19972-1-AP; 1:500 dilution) were purchased from ProteinTech Group, Inc. (Chicago, IL, USA). Immunohistochemistry results were quantified by measuring the positive stained cells. The positive staining was indicated at a density $>50 \%$.

Endothelial tube formation assay. The tube formation assay was performed using human umbilical vein endothelial cells (HUVECs; Type Culture Collection of the Chinese Academy of Sciences), which were cultured in RPMI-1640 supplemented with $20 \% \mathrm{FBS}$ at $37^{\circ} \mathrm{C}$ in an atmosphere containing $5 \% \mathrm{CO}_{2}$ as described previously (17). A total of $2 \times 10^{5}$ cells/well were seeded into 12 -well culture plates precoated with growth factor-reduced Matrigel ${ }^{\circledR}$ (BD Biosciences, Franklin Lakes, NJ, USA). Cells were treated with $5 \mathrm{nM}$ triptolide for $48 \mathrm{~h}$ and cells treated with si-VEGF siRNAs were employed as a positive control. After a $24-\mathrm{h}$ incubation at $37^{\circ} \mathrm{C}$, tube formation was imaged and quantified using an inverted microscope.

Bromodeoxyuridine (BrdU) incorporation assay. For the BrdU incorporation assay, HUVECs were pretreated with $5 \mathrm{nM}$ triptolide or $10 \mathrm{nM}$ siRNAs and cultured for $48 \mathrm{~h}$ at $37^{\circ} \mathrm{C}$. Cells were then processed for BrdU incorporation using a BrdU cell proliferation assay Kit (cat. no. K306-200; Biovision, Milpitas, CA, USA) according to the manufacturer's protocol, which has been described previously (17). Representative images were then captured using an inverted microscope and the number of cells were determined cells with fluorescence in five random fields and compared these with the control group.

Statistical analysis. Statistical analysis was performed using an unpaired Student's t-test. SPSS software (version 21.0; SPSS, Inc., Chicago, IL, USA). Results from in vitro experiments are presented at the mean \pm standard error of the mean from at least three experiments. In vivo results are presented as the mean \pm standard deviation. $\mathrm{P}<0.05$ was considered to indicate a statistically significant difference.

\section{Results}

Triptolide suppresses VEGFA expression. It has been reported that triptolide induces apoptosis and exhibits toxic effects on breast cancer cells (18); however, the function of triptolide in angiogenesis remains unclear. The expression of VEGFA, a key angiogenic activator, at the transcript level in six breast cancer cell lines was determined in the present study using semi-quantitative PCR (Fig. 1A). This revealed that VEGFA was relatively highly expressed in Hs578T and MDAMB231 cells compared with BT474, SKBR3, MCF7 and T47D cells. Next, Hs578T and MDAMB231 cells were treated with $5 \mathrm{nM}$ triptolide, which significantly reduced VEGFA mRNA levels after 48 and $72 \mathrm{~h}$ in a time-dependent manner (all $\mathrm{P}<0.05$; Fig. 1B). Western blotting was employed to confirm this observation at the protein level. In addition, the two cell lines were treated with different concentrations of triptolide ranging from 5-20 nM. Treatment with 10 and $20 \mathrm{nM}$ triptolide significantly reduced the expression of VEGFA mRNA 
A

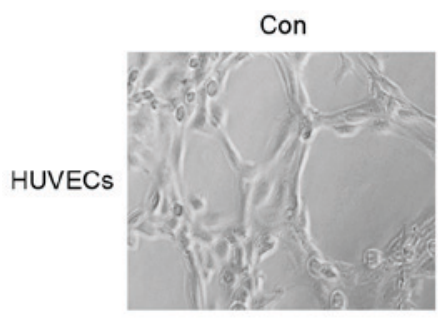

NC

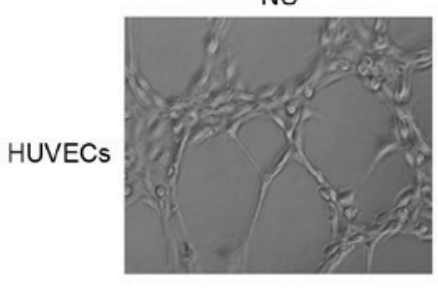

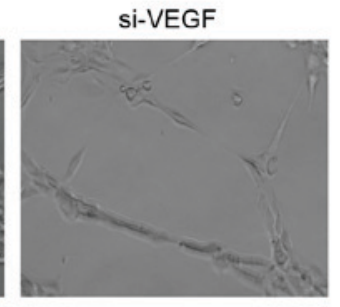

B
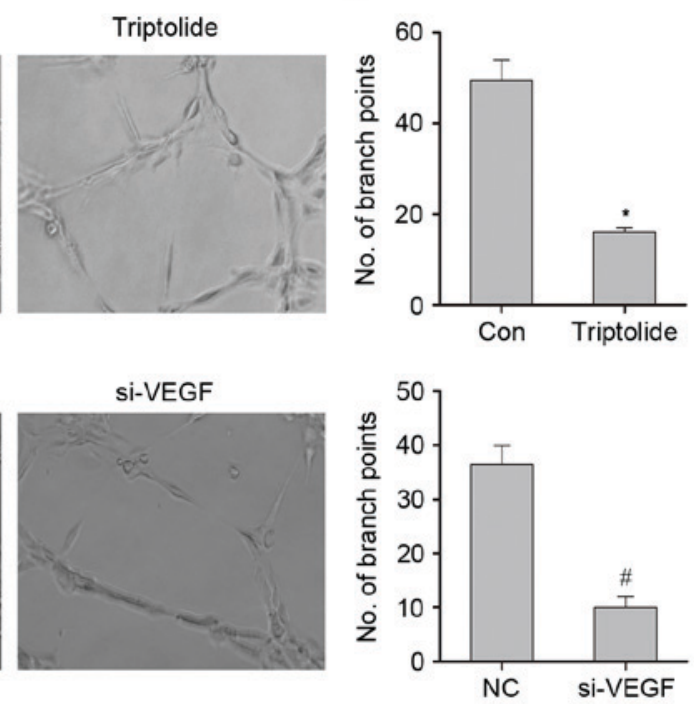

C
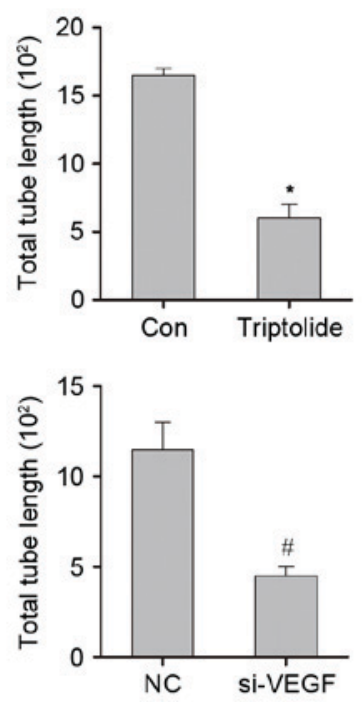

D
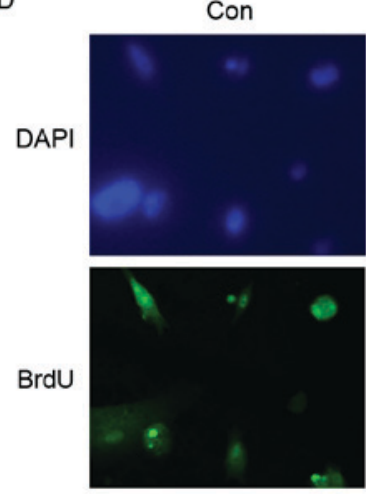

Triptolide
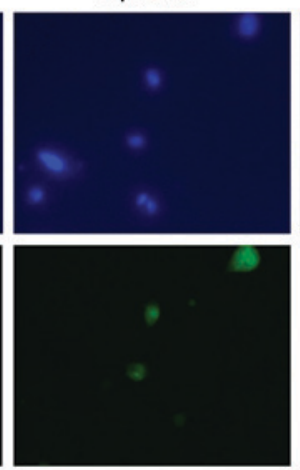

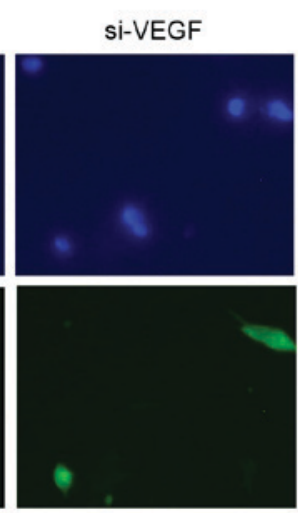

$E$

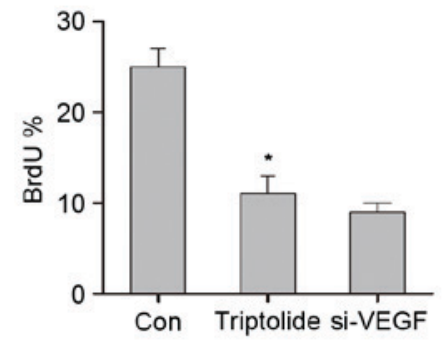

Figure 2. Triptolide inhibits endothelial tube formation and cell proliferation in vitro. (A) Representative images of the HUVEC tube formation assay after triptolide treatment (magnification, x200). Cells treated with si-VEGFA group were used as a positive control. The results were quantified by measuring (B) branch points and (C) total tube length. BrdU incorporation assay (D) representative images (magnification, $\mathrm{x} 200$ ) and (E) statistical analysis. ${ }^{*} \mathrm{P}<0.05$ vs. the Con group; ${ }^{\#} \mathrm{P}<0.05$ vs. the NC group. HUVEC, human umbilical vein endothelial cell; VEGFA, vascular endothelial growth factor A; BrdU, bromodeoxyuridine; Con, control; NC, negative control; si-VEGFA, small interfering RNA targeting VEGFA.

in a concentration-dependent manner (all $\mathrm{P}<0.05$; Fig. 1C). This was again confirmed at the protein level via western blot analysis. These results indicate that triptolide suppresses VEGFA expression in breast cancer cells.

Triptolide inhibits endothelial tube formation and cellular proliferation. To further address the impacts of triptolide in regulating VEGFA expression, HUVECs were used to evaluate the function of triptolide in angiogenesis. A previous study has indicated that triptolide exhibits toxic effects on these highly metastatic cells (18). In the present study, HUVECs were treated with $5 \mathrm{nM}$ triptolide for $48 \mathrm{~h}$, which induced less apoptosis (data not shown) than described in a previous study (18). As shown in Fig. 2A, the tube networks formed by HUVECs were more extensive in the control group compared with the triptolide group. Subsequently, siRNA targeting VEGFA was used as a positive control to directly decrease VEGFA expression, which revealed that the suppressive effect of triptolide on the tube networks formed by HUVECs was comparable with that of the HUVECs treated with the siRNA (Fig. 2A). These results were quantified by measuring the branch points (Fig. 2B) and total tube lengths (Fig. 2C). The data indicated that triptolide inhibits the tube formation of HUVECs. The effect of triptolide on endothelial cell proliferation was measured using the BrdU incorporation assay. Cells treated with triptolide or VEGFA-targeting siRNA exhibited decreased BrdU incorporation compared with the control group (Fig. 2D and E). These data suggest that triptolide inhibits endothelial cell proliferation and tube formation.

Triptolide inhibits angiogenesis. To analyze the effect of triptolide on breast cancer angiogenesis in vivo, MDAMB231 cells were injected into the mammary fat pads of female nude mice, which were subsequently treated with triptolide. After 6 weeks, the mice were sacrificed and tumor samples were extracted. Tumor microvasculature formation in the triptolide treatment group compared with the control group was investigated via immunohistochemistry. As shown in Fig. 3A, this revealed that the expression of the proangiogenic factor VEGFA was significantly decreased in the triptolide group compared with the control group $(\mathrm{P}<0.05)$. Furthermore, immunohistochemical staining for $\mathrm{CD} 31$ revealed that intrametastatic vasculature 
A

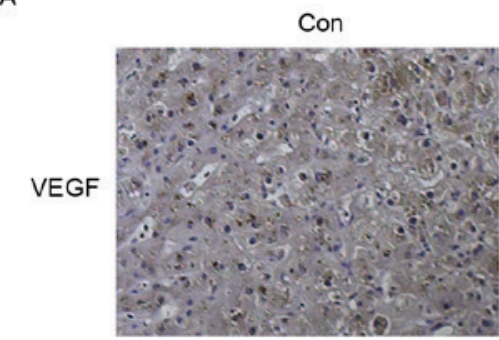

B

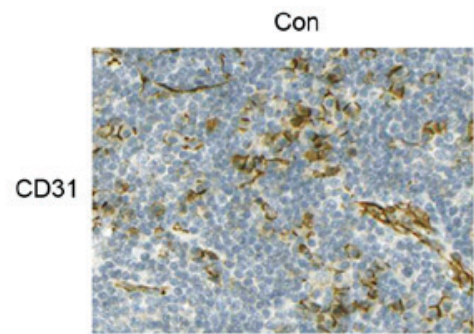

C

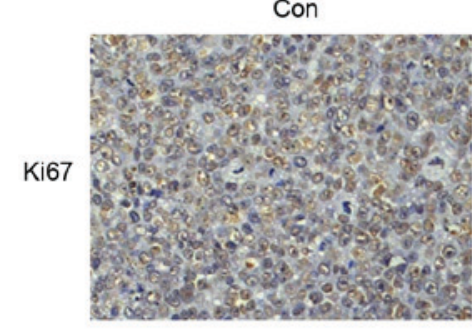

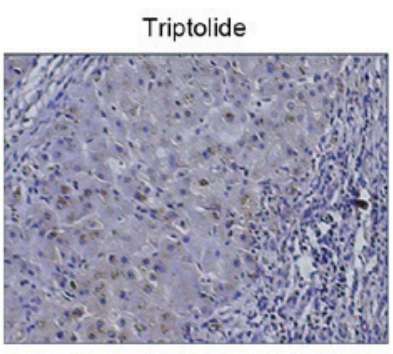
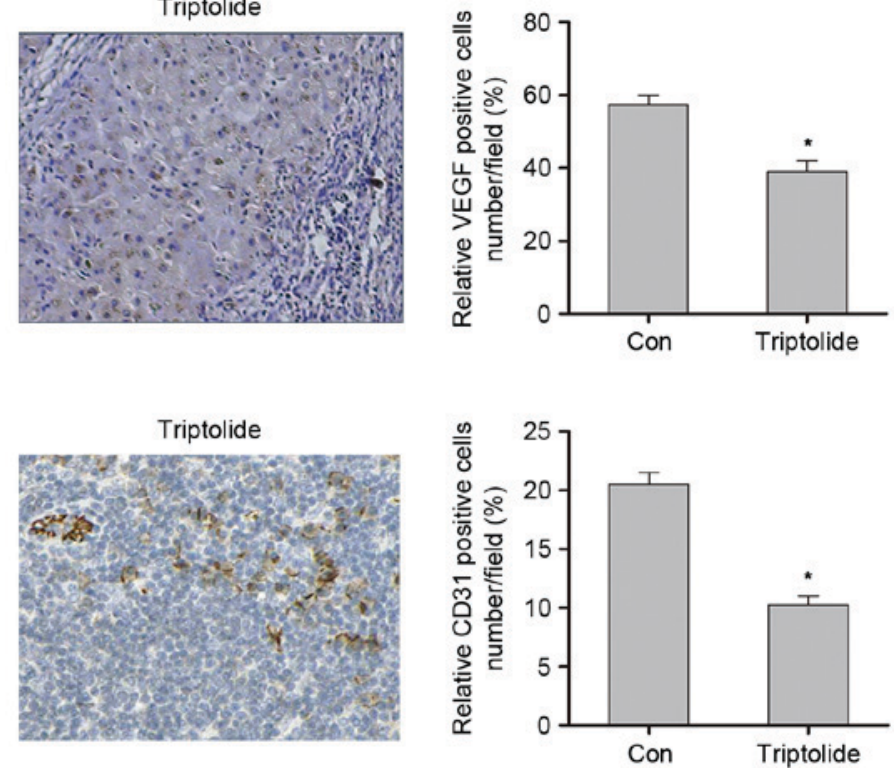

Triptolide
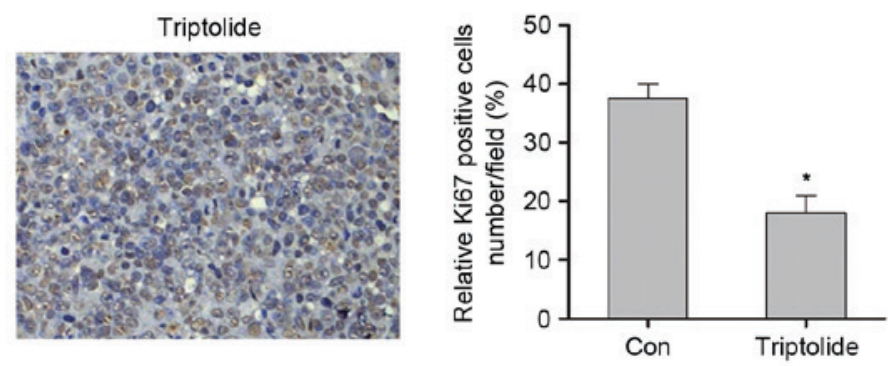

Figure 3. Triptolide inhibits angiogenesis in vivo. Tumor sections were immunohistochemically analyzed for the expression of VEGFA, CD31 and Ki67. Representative images (magnification, x100) and statistical analysis of (A) VEGFA, (B) CD31 and (C) Ki67 staining. "P<0.05 vs. the Con group. VEGFA, vascular endothelial growth factor A; CD31, cluster of differentiation 31; Ki67, anti-proliferation marker protein Ki67; Con, control.

A

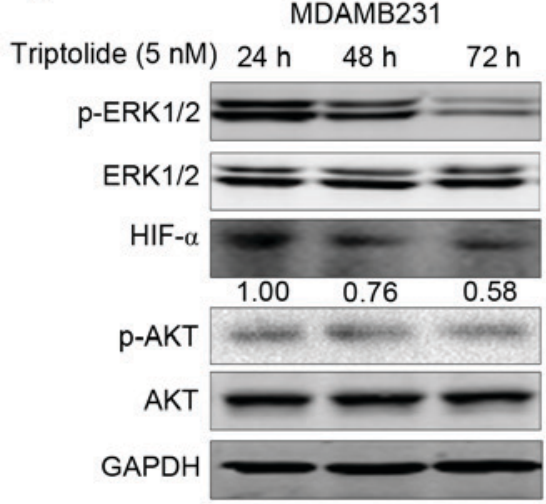

B

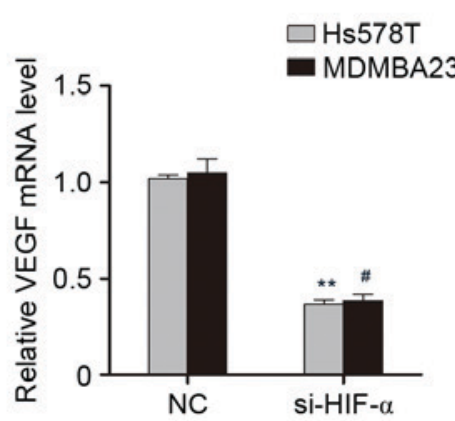

C

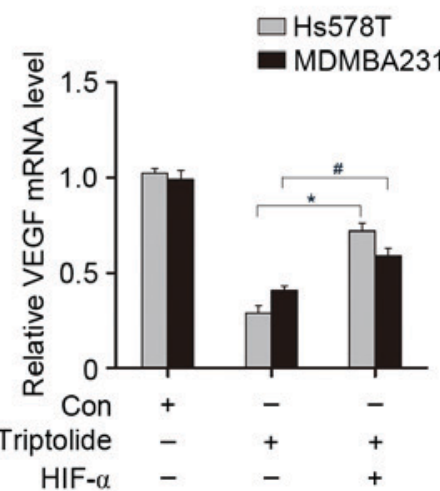

Figure 4. Triptolide regulates VEGFA expression through suppressing HIF1- $\alpha$ expression by inhibiting ERK1/2 activity. (A) Western blot analysis of ERK1/2 and Akt phosphorylation status, and HIF1- $\alpha$ expression, in MDAMB231 beast cancer cells treated with triptolide. (B) VEGFA expression was examined by RT-qPCR after knocking down HIF1- $\alpha$ expression. (C) VEGFA expression was examined by RT-qPCR after overexpression of HIF1- $\alpha$ and triptolide treatment. ${ }^{*} \mathrm{P}<0.05,{ }^{* *} \mathrm{P}<0.01$ vs. Hs578T cells at $24 \mathrm{~h} ;{ }^{\prime \prime} \mathrm{P}<0.05$ vs. MDAMB231 cells at $24 \mathrm{~h}$. VEGFA, vascular endothelial growth factor A; ERK1/2, extracellular signal-related kinase 1/2; HIF-1 $\alpha$, hypoxia inducible factor 1- $\alpha$; Akt, RAC- $\alpha$ serine/threonine-protein kinase; Con, control; si-HIF-1 $\alpha$, small interfering RNA targeting HIF-1 $\alpha$; RT-qPCR, reverse transcription-quantitative polymerase chain reaction.

was significantly decreased in the triptolide treatment group compared with the control group ( $\mathrm{P}<0.05$; Fig. $3 \mathrm{~B})$. In addition, a significant suppression in tumor cells proliferation was observed in the triptolide treatment group compared with the control group, as indicated by Ki67 staining ( $\mathrm{P}<0.05$; Fig. 3C). These data indicate that triptolide inhibits tumor angiogenesis. 
Triptolide regulates VEGFA expression through suppressing HIF1- $\alpha$ expression by inhibiting ERK1/2 activity. To further investigate the mechanism by which triptolide suppresses VEGFA expression, the Akt and mitogen-activated protein kinase signaling pathways, which have been in implicated in regulating VEGFA expression in breast cancer tumourigenesis (19), were examined. This demonstrated that triptolide suppressed ERK1/2 phosphorylation, while it had little effect on Akt phosphorylation (Fig. 4A). HIF1- $\alpha$ expression, which is regulated by ERK1/2, was also decreased in breast cancer cells treated with triptolide (Fig. 4A). As HIF1- $\alpha$ is key transcriptional regulator of VEGFA, knockdown of HIF1- $\alpha$ significantly suppressed VEGFA expression $(\mathrm{P}<0.05$; Fig. 4B). To determine whether HIF1- $\alpha$ was associated with the suppressive effect of triptolide on VEGFA expression, MDAMB231 and Hs578T cells were treated with triptolide followed by overexpression of HIF1- $\alpha$, after which VEGFA expression was examined. As shown in Fig. 4C, the expression of VEGFA was significantly upregulated following triptolide treatment and HIF1- $\alpha$ overexpression compared with triptolide treatment alone $(\mathrm{P}<0.05)$. These data suggest that the regulation of VEGFA expression by triptolide is partially dependent on the suppression of HIF1- $\alpha$ expression by inactivation of the ERK1/2 signaling pathway.

\section{Discussion}

It has been reported that triptolide has multiple functions, including immunosuppression and anti-inflammation (20-22). Recently, the in vivo inhibition of interleukin (IL)-1 $\beta$ by triptolide was examined in a mouse model of ulcerative colitis (23). In addition, triptolide inhibits the growth and metastasis of solid tumors, including melanoma, bladder cancer and gastric cancer (24). Furthermore, a recent study identified that triptolide induced breast cancer cell apoptosis and had toxic effects on tumor stem cells by regulating the Wnt/ $\beta$-catenin signaling pathway (18). Thus, the present study aimed to further investigate the function of triptolide on tumor angiogenesis.

Hs578T and MDAMB231 breast cancer cells were used to examine the functions of triptolide due to their relatively high expression of VEGFA. Notably, it has been reported that Hs578T and MDAMB231 cells are more sensitive to epidermal growth factor (EGF) stimulation (25). The invasive abilities of Hs578T and MDAMB231 cells were increased after EGF treatment compared with MDAMB453 and T47D cells (25). Furthermore, in the present study triptolide was demonstrated to suppress VEGFA expression in a dose- and time-dependent manner. In addition, the results of the tube formation assay revealed that triptolide inhibited HUVEC proliferation and migration. These results led to the investigation of the effect of triptolide in breast cancer in vivo. In the MDAMB231 cell xenograft transplantation mouse model, triptolide inhibited VEGFA expression, as demonstrated by immunohistochemistry. Ki67 and CD31 staining of the tumor sections also suggested decreased cell proliferation and angiogenesis.

The results of western blot analysis in the present study indicate that triptolide suppresses ERK1/2 activation and inhibits HIF1- $\alpha$ expression in breast cancer cells. Triptolide has been demonstrated to regulate several key signaling pathways in tumor progression, including the phosphatidylinositol-4,5-bisphosphate 3-kinase/Akt/Serine/threonine-protein kinase mTOR, tumor necrosis factor (TNF)-related apoptosis-inducing ligand and nuclear factor (NF)- $\mathrm{B}$ signaling pathways $(26,27)$. The present study examined Akt phosphorylation following triptolide treatment and identified a slight decrease in p-Akt, although this decrease was not significant.

Previous studies have indicated that triptolide can inhibit the expression of inflammation-associated genes, including IL-6, TNF- $\alpha$, and the adhesion molecules intercellular adhesion molecule 1 and P-selectin, in HUVECs treated with lipopolysaccharide (LPS) (28). However, the expression of HIF1- $\alpha$ was increased when LPS and triptolide were combined (28). These observations may be due to the fact that the tumor cells used were transformed epithelial cells that already expressed a higher level of inflammatory factors and HIF1- $\alpha$ compared with endothelial cells. Other studies have demonstrated that LPS can induce the epithelial-mesenchymal transition of tumor cells through regulation of the $\mathrm{NF}-\kappa \mathrm{B}$ signaling pathway, and that triptolide reverses this effect (29-31). In the present study, an siRNA pool was used to knockdown HIF1- $\alpha$, which confirmed that VEGFA expression was decreased $(32,33)$. Then, HIF1- $\alpha$ was overexpressed and the cells were treated with triptolide. Consistent with the hypothesis that triptolide regulates VEGFA expression through suppressing HIF1- $\alpha$ expression by inhibiting ERK1/2 activity, the overexpression of HIF1- $\alpha$ upregulated VEGFA expression, even with triptolide treatment.

In conclusion, the results of the present study indicate that triptolide inhibits breast cancer cell proliferation and angiogenesis through regulating the ERK1/2-HIF1- $\alpha$-VEGFA axis. Thus, triptolide is a potential therapeutic strategy for the treatment of breast cancer that required further study.

\section{References}

1. Siegel RL, Miller KD and Jemal A: Cancer statistics, 2015. CA Cancer J Clin 65: 5-29, 2015.

2. Endogenous Hormones and Breast Cancer Collaborative Group; Key TJ, Appleby PN, Reeves GK, Travis RC, Alberg AJ, Barricarte A, Berrino F, Krogh V, Sieri S, et al: Sex hormones and risk of breast cancer in premenopausal women: A collaborative reanalysis of individual participant data from seven prospective studies. Lancet Oncol 14: 1009-1019, 2013.

3. Couch FJ, Hart SN, Sharma P, Toland AE, Wang X, Miron P, Olson JE, Godwin AK, Pankratz VS, Olswold C, et al: Inherited mutations in 17 breast cancer susceptibility genes among a large triple-negative breast cancer cohort unselected for family history of breast cancer. J Clin Oncol 33: 304-311, 2015.

4. Deryugina EI and Quigley JP: Tumor angiogenesis: MMPmediated induction of intravasation- and metastasis-sustaining neovasculature. Matrix Biol 44-46: 94-112, 2015.

5. Richert MM, Vaidya KS, Mills CN, Wong D, Korz W, Hurst DR and Welch DR: Inhibition of CXCR4 by CTCE-9908 inhibits breast cancer metastasis to lung and bone. Oncol Rep 21: 761-767, 2009.

6. Bono F, De Smet F, Herbert C, De Bock K, Georgiadou M, Fons P, Tjwa M, Alcouffe C, Ny A, Bianciotto M, et al: Inhibition of tumor angiogenesis and growth by a small-molecule multi-FGF receptor blocker with allosteric properties. Cancer Cell 23: 477-488, 2013.

7. Seftor RE, Hess AR, Seftor EA, Kirschmann DA, Hardy KM, Margaryan NV and Hendrix MJ: Tumor cell vasculogenic mimicry: From controversy to therapeutic promise. Am J Pathol 181: 1115-1125, 2012. 
8. Zhou J, Xi C, Wang W, Fu X, Jinqiang L, Qiu Y, Jin J, Xu J and Huang Z: Triptolide-induced oxidative stress involved with Nrf2 contribute to cardiomyocyte apoptosis through mitochondrial dependent pathways. Toxicol Lett 230: 454-466, 2014.

9. Liu J, Jiang Z, Xiao J, Zhang Y, Lin S, Duan W, Yao J, Liu C, Huang X, Wang T, et al: Effects of triptolide from Tripterygium wilfordii on ERalpha and 553 expression in two human breast cancer cell lines. Phytomedicine 16: 1006-1013, 2009.

10. Pigneux A, Mahon FX, Uhalde M, Jeanneteau M, Lacombe F, Milpied N, Reiffers J and Belloc F: Triptolide cooperates with chemotherapy to induce apoptosis in acute myeloid leukemia cells. Exp Hematol 36: 1648-1659, 2008.

11. Park SW and Kim YI: Triptolide induces apoptosis of PMA-treated THP-1 cells through activation of caspases, inhibition of NF- $\mathrm{KB}$ and activation of MAPKs. Int J Oncol 43: $1169-1175,2013$

12. Chen F, Gao X and Shilatifard A: Stably paused genes revealed through inhibition of transcription initiation by the TFIIH inhibitor triptolide. Genes Dev 29: 39-47, 2015.

13. Owa C, Messina ME Jr and Halaby R: Triptolide induces lysosomal-mediated programmed cell death in MCF-7 breast cancer cells. Int J Womens Health 5: 557-569, 2013.

14. Hu C, Huang F, Deng G, Nie W, Huang W and Zeng X: miR-31 promotes oncogenesis in intrahepatic cholangiocarcinoma cells via the direct suppression of RASA1. Exp Ther Med 6 : $1265-1270,2013$.

15. Li J, Zhao X, Wang D, He W, Zhang S, Cao W, Huang Y, Wang L, Zhou S and Luo K: Up-regulated expression of phospholipase C, $\beta 1$ is associated with tumor cell proliferation and poor prognosis in hepatocellular carcinoma. Onco Targets Ther 9: 1697-1706, 2016.

16. Livak KJ and Schmittgen TD: Analysis of relative gene expression data using real-time quantitative PCR and the 2(-Delta Delta C(T)) method. Methods 25: 402-408, 2001.

17. Kong W, He L, Richards EJ, Challa S, Xu CX, Permuth-Wey J, Lancaster JM, Coppola D, Sellers TA, Djeu JY and Cheng JQ: Upregulation of miRNA-155 promotes tumour angiogenesis by targeting VHL and is associated with poor prognosis and triple-negative breast cancer. Oncogene 33: 679-689, 2014

18. Shao H, Ma J, Guo T and Hu R: Triptolide induces apoptosis of breast cancer cells via a mechanism associated with the Wnt/ $\beta$-catenin signaling pathway. Exp Ther Med 8: 505-508, 2014.

19. Jiang BH and Liu LZ: PI3K/PTEN signaling in angiogenesis and tumorigenesis. Adv Cancer Res 102: 19-65, 2009.

20. Qiu D, Zhao G, Aoki Y, Shi L, Uyei A, Nazarian S, Ng JC and Kao PN: Immunosuppressant PG490 (triptolide) inhibits T-cell interleukin-2 expression at the level of purine-box/nuclear factor of activated T-cells and NF-kappaB transcriptional activation. J Biol Chem 274: 13443-13450, 1999.

21. Chen X, Murakami T, Oppenheim JJ and Howard OM: Triptolide, a constituent of immunosuppressive Chinese herbal medicine, is a potent suppressor of dendritic-cell maturation and trafficking. Blood 106: 2409-2416, 2005
22. Wang X, Zhang L, Duan W, Liu B, Gong P, Ding Y and Wu X Anti-inflammatory effects of triptolide by inhibiting the NF- $\mathrm{KB}$ signalling pathway in LPS-induced acute lung injury in a murine model. Mol Med Rep 10: 447-452, 2014.

23. Zhang H, Gong C, Qu L, Ding X, Cao W, Chen H, Zhang B and Zhou G: Therapeutic effects of triptolide via the inhibition of IL-1 $\beta$ expression in a mouse model of ulcerative colitis. Exp Ther Med 12: 1279-1286, 2016

24. Yang S, Chen J, Guo Z, Xu XM, Wang L, Pei XF, Yang J, Underhill CB and Zhang L: Triptolide inhibits the growth and metastasis of solid tumors. Mol Cancer Ther 2: 65-72, 2003

25. Koh MS and Moon A: Activation of H-Ras and Racl correlates with epidermal growth factor-induced invasion in Hs578T and MDA-MB-231 breast carcinoma cells. Biochem Biophys Res Commun 406: 25-29, 2011.

26. Kim SH, Kang JG, Kim CS, Ihm SH, Choi MG, Yoo HJ and Lee SJ: Synergistic cytotoxicity of BIIB021 with triptolide through suppression of $\mathrm{PI} 3 \mathrm{~K} / \mathrm{Akt} / \mathrm{mTOR}$ and $\mathrm{NF}-\kappa \mathrm{B}$ signal pathways in thyroid carcinoma cells. Biomed Pharmacother 83: 22-32, 2016.

27. Zhao X, Zhang Q and Chen L: Triptolide induces the cell apoptosis of osteosarcoma cells through the TRAIL pathway. Oncol Rep 36: 1499-1505, 2016.

28. Galvez G, Santander S, Raskin I and Baldeon M: Effects of triptolide on the expression of inflammatory markers in lipopolysaccharide-treated human endothelial cells (HUVEC). FASEB J 29: 789-781, 2015.

29. Liu L, Salnikov AV, Bauer N, Aleksandrowicz E, Labsch S, Nwaeburu C, Mattern J, Gladkich J, Schemmer P, Werner J and Herr I: Triptolide reverses hypoxia-induced epithelial-mesenchymal transition and stem-like features in pancreatic cancer by $\mathrm{NF}-\kappa \mathrm{B}$ downregulation. Int J Cancer 134: 2489-2503, 2014.

30. Sarkar TR, Battula VL, Werden SJ, Vijay GV, Ramirez-Peña EQ, Taube JH, Chang JT, Miura N, Porter W, Sphyris N, et al: GD3 synthase regulates epithelial-mesenchymal transition and metastasis in breast cancer. Oncogene 34: 2958-2967, 2015.

31. Huang T, Chen Z and Fang L: Curcumin inhibits LPS-induced EMT through downregulation of NF- $\kappa$ B-Snail signaling in breast cancer cells. Oncol Rep 29: 117-124, 2013.

32. Ahluwalia A and Tarnawski AS: Critical role of hypoxia sensor-HIF-1 $\alpha$ in VEGF gene activation. Implications for angiogenesis and tissue injury healing. Curr Med Chem 19: 90-97, 2012

33. Ke Q and Costa M: Hypoxia-inducible factor-1 (HIF-1). Mol Pharmacol 70: 1469-1480, 2006

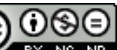

This work is licensed under a Creative Commons Attribution-NonCommercial-NoDerivatives 4.0 International (CC BY-NC-ND 4.0) License. 